\title{
Study of load history effects on the high cycle fatigue properties of high-strength low-alloy steel from self-heating measurements
}

\author{
Julien Louge ${ }^{1,2, *}$, Cédric Doudard ${ }^{1}$, Sylvain Calloch $^{1,}$ and Bastien Weber $^{2}$ \\ ${ }^{1}$ Institut de Recherche Dupuy de Lôme (FRE 3744), UBS/UBBO/ENSTA-Bretagne/ENIB - 2, rue François Verny 29806 Brest cedex 9 , \\ France \\ ${ }^{2}$ ArcelorMittal Maizières Research, Voie Romaine 57283 Maizières-lès-Metz cedex, France
}

\begin{abstract}
In the context of high cycle fatigue (HCF), the experimental characterization of the fatigue properties is often performed by using specimens in a virgin state (i.e., without preliminary loading), and with a constant stress amplitude for each specimen. However, the load history applied to a real structure is more complex and the fatigue life prediction remains a difficult task because of the time dedicated to the classical fatigue tests (i.e., the specimen is loaded until failure) and the dispersion of fatigue lives. The load history effects on the HCF properties is characterized using an alternative method: self-heating measurements under cyclic loadings. This method is based on the observation of the mean steady state temperature evolution of a specimen under a successive series of cyclic loadings with increasing stress amplitude for each loading series. A probabilistic two-scale model was developed from the self-heating method able to predict HCF properties. Some self-heating tests are performed to study the influence of a load history effects. It seems that the plasticity is the most influential factor. So, the evolution of the plasticity is observed at the surface of the material under cyclic loading. There is a significant evolution in function of the plastic pre-strain.
\end{abstract}

\section{Introduction}

The standard method to characterize high cycle fatigue properties of steel grade requires one month of tests. To reduce the time of characterization, some fast methods have been developed, such as the self-heating method. This method, associated with a two scales probabilistic model, allows to obtain the fatigue limit with only one specimen in less than one day. This kind of model assumes that the damage is mainly due to microplasticity activation. Many steel grades have been studied with the self-heating method [1,2]. To go deeper in this way, and to expand the two scales probabilistic model, two kind of load history are studied with the self-heating method: a plastic pre-straining and a cyclic-pre-loading.

First, the self-heating method and the two scales probabilistic model associated are presented. The heat conduction equation is reminded, the test procedure and heat acquisition are introduced. Then, the two kind of load history are studied to show their influence on the self-heating curves. Finally, the mechanisms strain are analysed by observing the emergence of plasticity at the surface of the material under cyclic loading. The material studied is a High Strength Low Alloyed steel HSLA320 $2 \mathrm{~mm}$ thick (bare product) which was hot rolled. The mechanical properties the rolling direction (direction of interest) are: Yield Stress $340 \mathrm{MPa}$, Tensile Strength $445 \mathrm{MPa}$ and a total elongation $31 \%$.

\section{Self-heating method}

\subsection{Self-heating test procedure}

The self-heating method consist on apply a successive series of cyclic loadings, with increasing the stress amplitude, $\Sigma_{0}$ (the amplitude is constant per series) (Fig.1). A 0D approach is chosen and the corresponding heat conduction equation is given by

$$
\dot{\theta}^{0 \mathrm{D}}+\frac{\theta^{0 \mathrm{D}}}{\tau^{0 \mathrm{D}}}=S_{t}^{0 \mathrm{D}},
$$

Fig.1. Successive series of cyclic loadings while increasing stress amplitudes used for self-heating tests. 


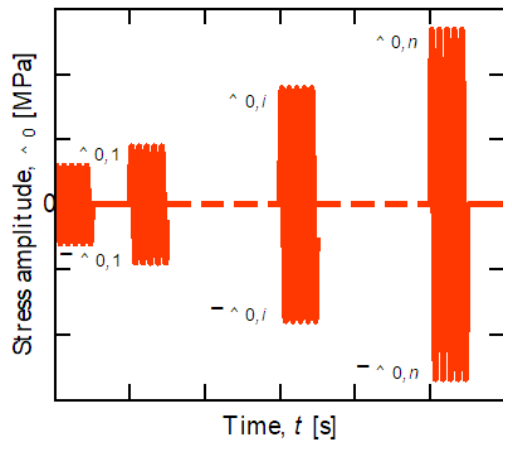

The temperature is measured by three thermocouples, one fixed on the center of the specimen and the two other are fixed on the grips of the hydraulic machine. The evolution of the mean temperature elevation during a series of cyclic loading is given by

$$
\theta^{0 \mathrm{D}}=T_{\text {specimen }}-\frac{T_{\text {uppergrip }}+T_{\text {lowergrip }}}{2},
$$

and is shown in (Fig.2). This evolution reached a steadystate, called $\bar{\theta}^{\text {OD }}$.

Fig.2. Evolution of the mean temperature elevation during one series of cyclic loadings.

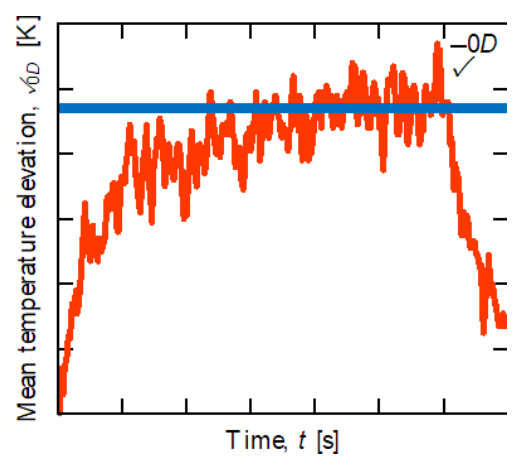

After all the series of cyclic loadings are performed on a specimen, the self-heating curve is obtained, representing the mean steady-state temperature evolution $\bar{\theta}^{0 \mathrm{D}}$ relating to the stress amplitude $\Sigma_{0}$ (Fig.3). Two selfheating regimes are observed. The first one for low amplitudes of load, called primary regime, and the second one for the highest amplitudes, called the secondary regime. It has been shown empirically that the intersection between the two self-heating regimes, in a logarithmic scale, provides a good approximation of the mean fatigue limit of the studied material $\bar{\Sigma}_{\infty}$.

Fig.3. Self-heating curves in a logarithmic scale.

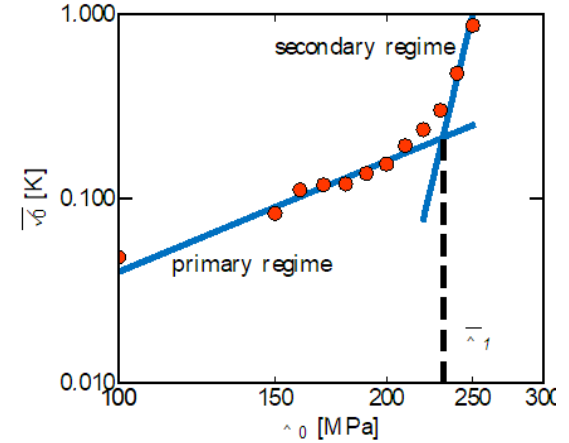

\subsection{Two scales probabilistic model}

To describe the self-heating curves and predict the fatigue properties, a two scales probabilistic model has been proposed [1-3]. A REV (Representative Elementary Volume) is defined as an elasto-platic matrix containing a set of inclusions with an additional plastic hardening (Fig.4). Under low amplitude of cyclic loadings, the dissipation is due to the elasto-plastic behaviour of the matrix. Then, above a given stress amplitude, the dissipation is due to the behaviour of the matrix combined with the activated inclusions.

Fig.4. Principle of the two scales probabilistic model.

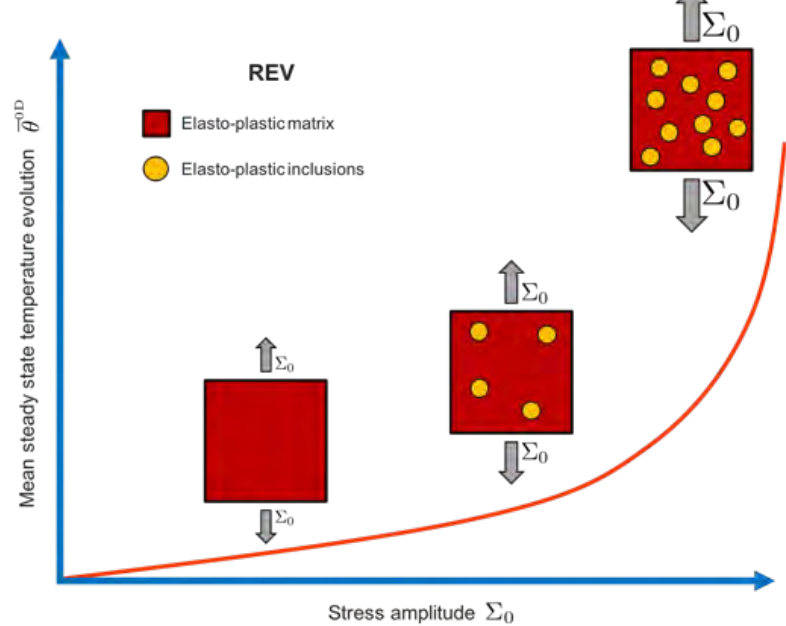

The dissipation associated with the matrix corresponds to the primary regime. The secondary regime is due to the dissipation of the matrix associated to the set of inclusions. Thus, the mean steady-state temperature elevation $\bar{\theta}^{0 \mathrm{D}}$ can be written as

$$
\bar{\theta}^{0 \mathrm{D}}=\alpha\left(\frac{\Sigma_{0}}{\Sigma_{\max }}\right)^{2}+\beta\left(\frac{\Sigma_{0}}{\Sigma_{\max }}\right)^{m+2},
$$

with the parameters $\alpha$ and $\beta$, which correspond respectively to the intensity of the primary and the secondary regime. The parameter $m$ corresponds to the 
slope of the secondary regime, related to the gradual appearance of active sites.

In the model, it is assumed that the site activation governs the fatigue damage initiation. Within the weakest link theory, the probability of failure, called $P_{F}$, is the probability of finding at least one active site. To determine the number of cycles to failure $N\left(P_{F}\right)$, an energetic criterion is used. The cyclic dissipation energy is constant for each cycle, so the critical dissipated energy can be written as a function of $N\left(P_{F}\right)$. Then, the number of cycle to failure is given by

$$
N=\frac{A \Sigma_{\max }^{2}}{\bar{\Sigma}_{\infty}\left(P_{F}\right)\left\langle\Sigma_{0}-\bar{\Sigma}_{\infty}\left(P_{F}\right)\right\rangle},
$$

with $\bar{\Sigma}_{\infty}\left(P_{F}\right)$ the fatigue limit associated with a given probability of failure. Finally, the ability of the model to predict S-N-P curves is shown Fig.5.

Fig.5. Validation of the method with an S-N curve.

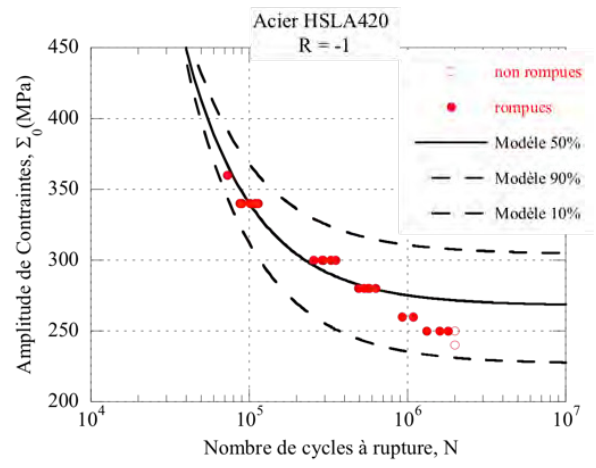

\section{Load history effects influence on the self-heating measurements}

The self-heating (SH) method has been developed [1-3] to characterize the fatigue properties using specimen in a as-delivered state (i.e., without preliminary loading). The first aim of this study is to analyse the influence of load history effects on HCF properties. For that, two loads history are studied: a plastic pre-strain range and an initial series of cyclic loading.

In order to observe the evolution of the self-heating curves after performed a load history, two indicators are introduced. The first one represents the evolution of the intensity of the primary regime, called $\alpha_{\text {norm }}$. It corresponds to the ratio between the amplitude value for the as-delivered state, $\alpha_{\text {virgin, }}$ and the value for the specimen where a history loading was applied, $\alpha_{\text {history }}$, given by

$$
\alpha_{\text {norm }}=\frac{\alpha_{\text {virgin }}}{\alpha_{\text {virgin }}}
$$

The parameter $\beta$ and the exponent $m+2$ (Eq.3) represent respectively the intensity and the slope of the secondary self-heating regime. The slope is determined for the as-delivered specimen, $m=23$, and it is considered to be identical for the other self-heating curves. It has been shown that the secondary regime can be related to the fatigue life properties. So, the evolution of the secondary regime intensity corresponds directly to the evolution of the fatigue limit $\bar{\Sigma}_{\infty}$, estimated by the selfheating method. So, the second indicator, called $\beta_{\text {norm }}$ given by

$$
\beta_{\text {norm }}=\frac{\bar{\Sigma}_{\infty}^{\text {history }}}{\bar{\Sigma}_{\infty}^{\text {virgin }}}=\left(\frac{\beta_{\text {virgin }}}{\beta_{\text {history }}}\right)^{1 / m},
$$

represents the ratio between the fatigue limit for the asdelivered state, $\bar{\Sigma}_{\infty}^{\text {virgin }}$, and the fatigue limit for the specimen where an history loading was applied, $\bar{\Sigma}_{\infty}^{\text {history }}$, determined by the self-heating method. For example, a value 0.95 for this indicator corresponds to a $5 \%$ decrease of the fatigue limit.

\subsection{Plastic pre-strain effect}

In order to study a plastic pre-strain effect on the selfheating measurements, a tensile test is performed on a specimen until a target strain level is reached. Then, a self-heating test is carried out (Fig.6).

The result of the self-heating test for a plastic prestrain level of $1 \%$ is shown in Fig.7. The amplitude of the primary regime increased. It is not so easy to appreciate the evolution of the secondary regime in this figure, but the evolution of the parameters is given in Tab.1. The parameter $\beta_{\text {norm }}$ is equal to 0.94 , so the fatigue limit for the pre-strain material is around $6 \%$ lower compared to the as-delivered state.

Fig. 6. Principle of the test performed to study the influence of a plastic pre-strain effect.

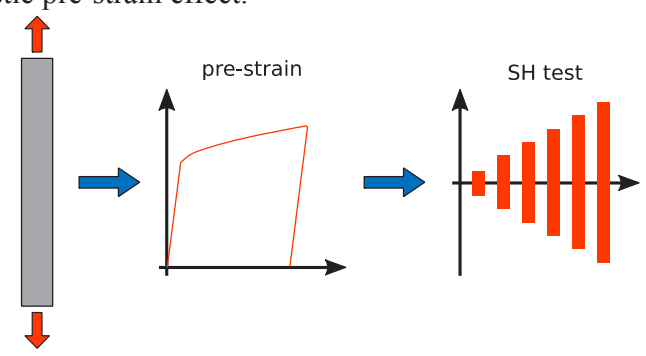

Fig. 7. Comparison of the self-heating curves for the virgin state and the $1 \%$ level of plastic pre-strain state. 


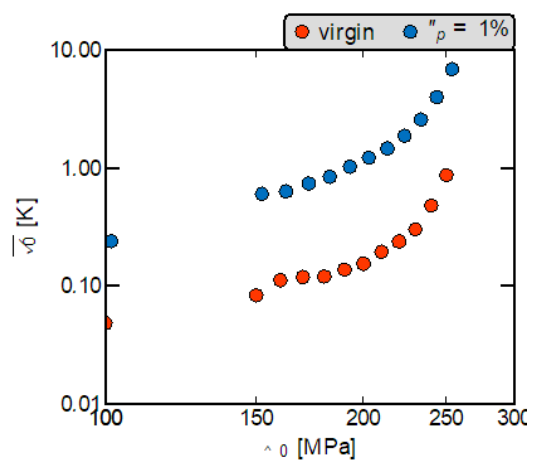

Tab. 1. Values of the model parameters for the as-delivered state and the $1 \%$ level of plastic pre-strain state.

\begin{tabular}{|c|c|c|c|c|}
\hline State & $\alpha[\mathrm{K}]$ & $\beta[\mathrm{K}]$ & $\alpha_{\text {norm }}[-]$ & $\beta_{\text {norm }}[-]$ \\
\hline as-delivered & 0.251 & 0.696 & 1.00 & 1.00 \\
\hline Pre-strain 1\% & 1.558 & 5.048 & 6.200 & 0.924 \\
\hline
\end{tabular}

\subsection{Initial series of cyclic loading effect}

For this study, an initial series of cyclic loadings is applied to the specimen, then a self-heating test is performed (Fig.8). First, the influence of the stress amplitude loading is investigated for a constant number of cycles ( $\mathrm{N}=50000$ cycles). Then, the influence of the cycle number is analysed for a constant stress amplitude $\Sigma_{0}=210 \mathrm{MPa}$ (transition between the primary and the secondary regime).

Fig. 8. Principle of the test performed to study the influence of an initial series of cyclic loading.

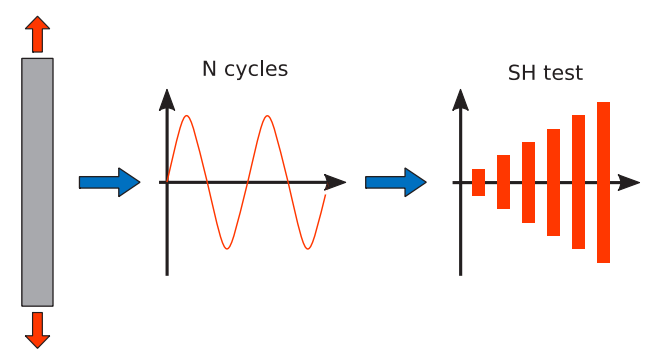

\subsubsection{Influence of the stress amplitude}

A substantial evolution of the self-heating curves can be observed for the different stress amplitudes (Fig.9). However, the maximum temperature elevation is lower than the elevation obtained previously with $1 \%$ of plastic pre-strain.
Fig. 9. Influence of the stress amplitude of a cyclic pre-loading on self-heating curves, with $\mathrm{N}=50000$ cycles.

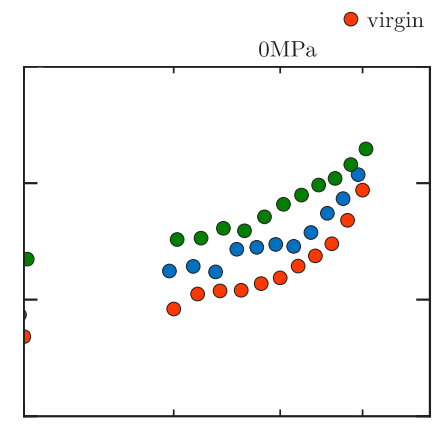

The evolution of the two parameters $\alpha$ and $\beta$, as well as the normalized parameters $\alpha_{\text {norm }}$ and $\beta_{\text {norm }}$ are shown in Tab.2. This history loading seems to have less impact than a plastic pre-strain. Indeed, with only $1 \%$ of plastic pre-straining, the primary regime intensity is multiplied by around 5 whereas a cyclic pre-loading with $\Sigma_{0}=240 \mathrm{MPa}$ leads to a factor 1.5 . The fatigue limit varies very slightly.

Tab. 2. Influence of the stress amplitude of a cyclic preloading on the model's parameters, with $\mathrm{N}=50000$ cycles.

\begin{tabular}{|c|c|c|c|c|}
\hline State & $\alpha[\mathrm{K}]$ & $\beta[\mathrm{K}]$ & $\alpha_{\text {norm }}[-]$ & $\beta_{\text {norm }}[-]$ \\
\hline as-delivered & 0.251 & 0.696 & 1 & 1 \\
\hline 50000 cycles & 0.482 & 1.168 & 1.918 & 0.980 \\
$210 \mathrm{MPa}$ & & & & \\
\hline $50000 \mathrm{cycles}$ & 0.856 & 1.568 & 3.405 & 0.968 \\
$240 \mathrm{MPa}$ & & & & \\
\hline
\end{tabular}

\subsubsection{Influence of the number of cycles}

Fig. 10. Influence of the number of cycles on self-heating curves after a cyclic pre-loading, with $\Sigma_{0}=210 \mathrm{MPa}$.

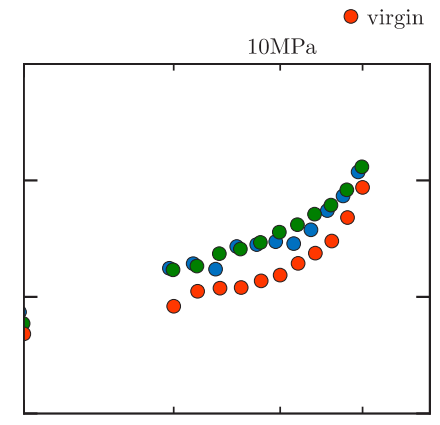

The chosen values to study the influence of the number of cycles are 50000 and 500000 cycles, for the same stress amplitude $\Sigma_{0}=210 \mathrm{MPa}$. The results show 
that the self-heating curves are of the same order of magnitude as previously (Fig.10). The indicators show that the both self-heating regimes are almost no affected by the number of cycles (Tab.3).

Tab. 3. Influence of the number of cycles on the model's parameters after a cyclic pre-loading, with $\Sigma_{0}=210 \mathrm{MPa}$.

\begin{tabular}{|c|c|c|c|c|}
\hline State & $\alpha[\mathrm{K}]$ & $\beta[\mathrm{K}]$ & $\alpha_{\text {norm }}[-]$ & $\beta_{\text {norm }}[-]$ \\
\hline as-delivered & 0.251 & 0.696 & 1 & 1 \\
\hline $\begin{array}{c}50000 \text { cycles } \\
210 \mathrm{MPa}\end{array}$ & 0.482 & 1.168 & 1.918 & 0.980 \\
\hline $\begin{array}{c}500000 \text { cycles } \\
210 \mathrm{MPa}\end{array}$ & 0.531 & 1.058 & 2.114 & 0.984 \\
\hline
\end{tabular}

\subsection{Combined effects of a plastic pre-straining and an initial cyclic loading}

To show which history loading (pre-straining or precycling) has the most influence, a $1 \%$ of plastic prestraining is performed on a specimen and then a cyclic pre-loading with $\mathrm{N}=50000$ cycles and $\Sigma_{0}=210 \mathrm{MPa}$ is applied. Finally, a self-heating test is carried out (Fig.11). The self-heating curves are provided in Fig.12.

Fig. 11. Principle of the test performed to study the combined effects of a plastic pre-straining and an initial cyclic loading.

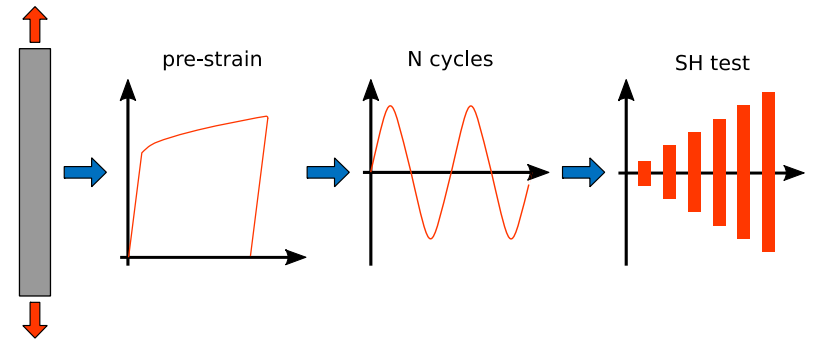

Fig. 12. Self-heating curves obtained for $1 \%$ of plastic prestrain range and with or without a cyclic pre-loading $\left(\mathrm{N}=50000\right.$ cycles at $\left.\Sigma_{0}=210 \mathrm{MPa}\right)$.

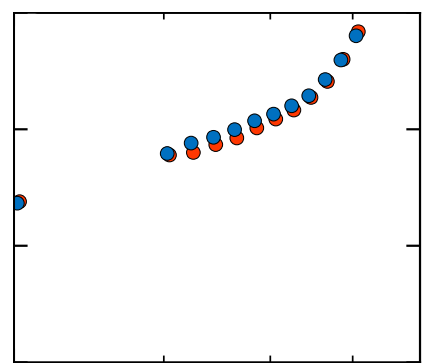

Tab. 4. Model's parameters obtained for $1 \%$ of plastic prestrain range and with or without a cyclic pre-loading $\left(\mathrm{N}=50000\right.$ cycles at $\left.\Sigma_{0}=210 \mathrm{MPa}\right)$.

\begin{tabular}{|c|c|c|c|c|}
\hline State & $\alpha[\mathrm{K}]$ & $\beta[\mathrm{K}]$ & $\alpha_{\text {norm }}[-]$ & $\beta_{\text {norm }}[-]$ \\
\hline Pre-strain 1\% & 1.558 & 5.048 & 1.000 & 1.000 \\
\hline $\begin{array}{c}\text { Pre-strain 1\% } \\
50000 \text { cycles } \\
210 \mathrm{MPa}\end{array}$ & 1.990 & 4.738 & 1.227 & 1.002 \\
\hline
\end{tabular}

Here, to determine the parameters $\alpha_{\text {norm }}$ and $\beta_{\text {norm }}$, the reference state is no longer the as-delivered one, but the material pre-strained at $1 \%$ of plastic strain (Tab.4).

The main conclusion is that after a plastic prestraining, the self-heating curve is almost the same with performing an initial series of cyclic loading.

\subsection{Conclusions}

In this study, it has been shown that a plastic pre-strain range has an important impact on the self-heating curves. However, an initial series of cyclic loading has a slightly influence and this influence can explain the difference between the fatigue limit determined from a two blocks fatigue test. Indeed, if a such as fatigue test is performed with the first block amplitude higher than the second one, the fatigue limit determined is lower than if the two blocks are inverted. For the modelling, the microplasticity would be govern the fatigue properties.

\section{Observation of the deformation mechanism}

For a self-heating test, the temperature elevation is due to dissipative mechanisms which occur in the volume of the material. It has been shown in the previous part that these mechanisms are caused by the micro-plastic strain. This micro-plasticity is represented by activated inclusions in the two scales probabilistic model and the activation evolution of these inclusions is given by

$$
\lambda=\frac{1}{V_{0}}\left(\frac{\Sigma_{0}}{s_{0}}\right)^{m},
$$

with $\lambda$ the activated inclusions density, $V_{0}$ and $S_{0}{ }^{m}$ material parameters.

The evolution of this micro-plasticity can be observed at the surface of the specimen by means of optical microscope. The procedure is explained hereafter and in [1]. These observations allow to justify the form of the equation (Eq.7).

\subsection{Experimental protocol}

\subsubsection{Specimens}


The shape of used specimens has a varying cross-section so that the studied mechanisms are localized in its center (Fig.13).

Fig. 13. Shape of the used specimens to observe deformation mechanisms.

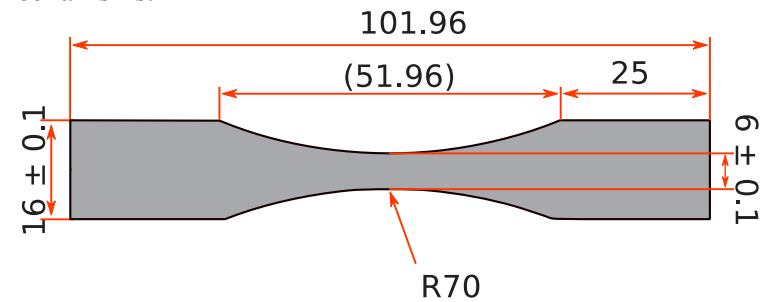

Fig. 14. Principle to apply uniform plastic pre-strain on the specimen.

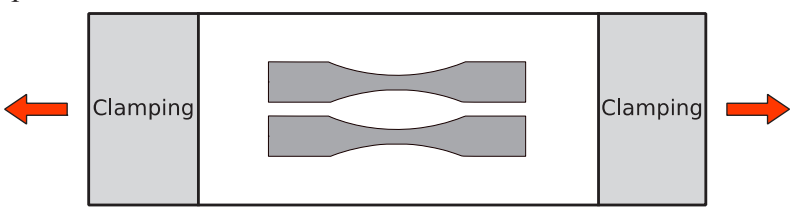

The material surface has its initial roughness. To observe the deformation mechanism at the face of the specimen, a mechanic follows by an electrolytic polishing were applied. This last polishing is achieved at the center of the specimen and has many advantages. Indeed, the repeatability of the surface state is guaranteed, the residual stress induced by the mechanical polishing is vanished and the microstructure is revealed.

Plastic straining was applied to a prismatic coupon and the specimens extracted by electro-discharge machining (Fig14).

\subsubsection{Loading}

Interrupted fatigue test was performed by block of $N_{\text {block }}$ cycles at a constant stress amplitude, $\Sigma_{O}$, until $80 \%-90 \%$ of the number of cycles to failure, $N_{\mathrm{r}}$ (Fig.15). The initial picture is taken before any load is applied (i.e. $\mathrm{N}=0$ cycle). Then, after each block of $N_{\text {block }}$ cycles another picture is taken and so on. This particular protocol is applied for several stress amplitude and different initials states of material (as-delivered or with an initially plastic pre-strained).

Fig. 15. Principle of the interrupted fatigue test to observe the deformation mechanisms at the surface of the material.

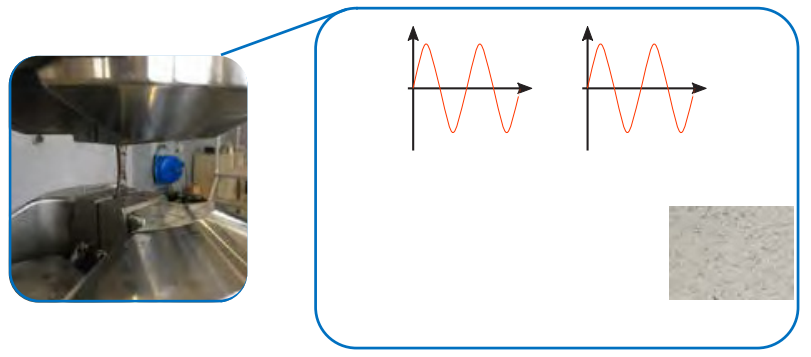

\subsubsection{Observations}

The pictures are taken by means of an optical microscope at magnification factor $\mathrm{x} 1000$. After cyclic loading with stress amplitude below the macroscopic elastic limit, some "dark stains" are observed (Fig.16). It has shown that these "dark stains" corresponding to PSB (Persistent Slip Bands) [1,4] (Fig.17). These PSB demonstrate the occurence of micro-plasticity in the material. From these pictures, the number and the spatial distribution of PSB can be analysed.

Fig. 16. Evolution of the PSB occurence under cyclic loading at $\Sigma_{O}=260 \mathrm{MPa}$.

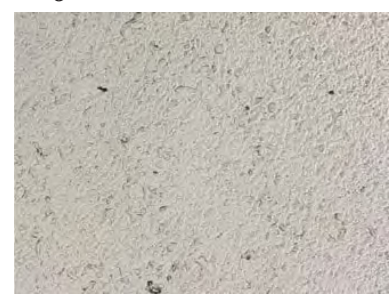

(a) Initial picture: 0 cycle

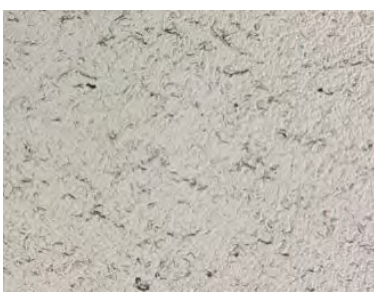

(b) Final picture: 100000 cycles
Fig. 17. Observation of the PSB at the surface of the material and representation of the mechanisms.

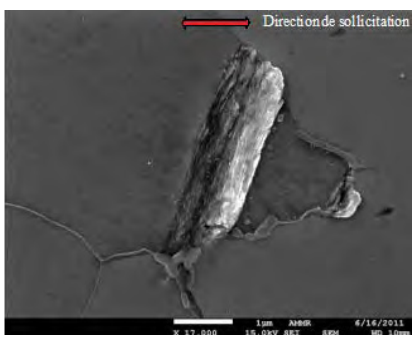

(a) SEM observation

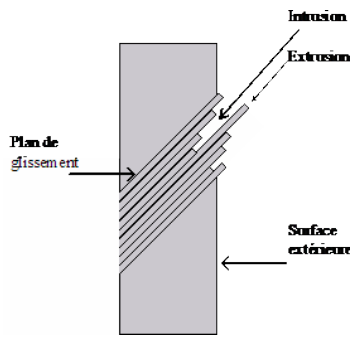

(b) Representation of the PSB

\subsection{Results}

This experimental protocol is applied for several stress amplitudes. Two states of the material are studied: the as-delivered state and the 1\% plastic pre-strain (Fig.18). These results show that a plastic pre-straining has an influence on the occurence of the PSB, so the evolution of the plasticity into the material. With a $1 \%$ plastic prestrain, the emergence of PSB appears for lower stress amplitude than for the as-delivered state. So, for the same stress amplitude, the dissipation is most important for the pre-strained state, which is consistent with the self-heating results.

Fig. 18. Evolution of the number of the PSB for different stress amplitude under cyclic loading. 


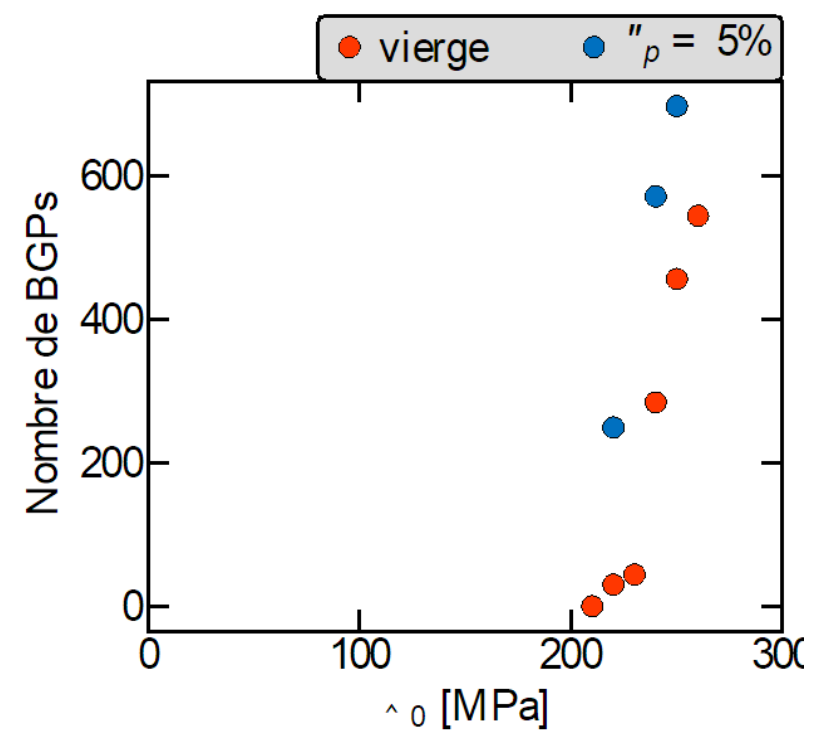

\section{Conclusions}

The goal of this study was to study the influence of load history effects on the self-heating measurements under cyclic loading. On the one hand, it has shown that the load history effects impacted the material response (without question the presence of the two self-heating regimes). On the other hand, an analyse combining two load history effects was proposed if the initial state is defined be the plasticity level reached during the preload (either a plastic pre-strain or an initial loading).

Then, to validate the interpretation of the previous result (e.g., presence of both self-heating regimes) an experimental protocol was developed to study the emergence of microplasticity under cyclic loadings at the surface of a specimen. These observations are in accordance with the evolution of the secondary regime of the self-heating curves.

The next step consists on developing a particular model to describe these results: the probabilistic twoscale model developed by $[1,2]$ have to be extended to take into account this load history effects on fatigue properties.

\section{References}

1. R. Munier, PhD thesis, (2012)

2. R. Munier, C. Doudard, S. Calloch, B. Weber, Int J

Fatigue, 63, 46-61, (2014)

3. C. Doudard, PhD thesis, (2004)

4. S. Suresh, Fatigue of Materials, (1991) 